behaviour due to increased screen time and decreased in-person sexual activity. This analysis describes the prevalence and factors associated with virtual sex before and during COVID19 measures in Panama.

Methods An online survey conducted among $\geq 18$ years individuals residing in Panama using social media recruitment, from August 8-September 12, 2020, at the end of strict lockdown measures. Questions included demographics, virtual sex (sexting [sharing/receiving nude/semi-nude photos/video] and cybersex [sexual acts in front of a camera] three months before and during COVID-19-measures. Logistic regression was used to identify associations with increased use of virtual sex.

Results Overall, 960 individuals participated; 526 (54.8\%) identified as cis-women, $366(31.1 \%)$ as cis-men, and 68 $(7.1 \%)$ as non-binary/another gender; median age was $28 \mathrm{y}$ (IQR:23-37y). Before COVID-19-measures, 44.1\% (369/837) reported sexting, 20.4\% (172/842) cybersex, 46.4\% (392/485) virtual sex. During COVID-19-measures, sexting, cybersex and virtual sex increased for 17.4\%(139/797), 9.4\%(74/790), and $19.9 \%(159 / 800)$ of participants, respectively. More cis-men reported virtual sex increase than cis-women $(25.7 \%$ vs $17.2 \%$ [rural/urban adjusted]AOR=1.69, 95\%CI:1.18-2.43). Bisexual $(38.7 \%$ [gender and urban/rural adjusted]AOR $=2.08,95 \%$ CI:1.09-3.95) and lesbian/gay participants $(42.4 \%, \mathrm{AOR}=2.64$, 95\%CI:1.47-4.73) reported virtual sex increase more frequently compared to heterosexual participants (16.1\%). Increase in casual sex was associated with increase in virtual sex $(45.0 \%$ vs less casual sex 25.3\%, AOR=4.06, 95\% CI:1.24-13.35). Increased pornography use was associated with increased virtual sex $(52.0 \%$ vs $7.8 \%$ decreased pornography, $\mathrm{AOR}=5.68,95 \% \mathrm{CI}: 2.40-13.44)$. Among participants with a long-term partner, virtual sex increased among those who reported more partnership conflicts during than before COVID-19-measures $(27.8 \%$ vs $12.8 \%$ among those who reported fewer conflicts, $\mathrm{AOR}=2.88,95 \% \mathrm{CI}: 1.45-5.72$ ).

Conclusions Virtual sex was common before COVID-19-measures in Panama. During COVID-19-measures, virtual sex increased among cis-men, lesbian/gay and bisexual participants. Virtual sex was associated with increased pornography use, casual sex, and increased conflicts with long-term partners.

\section{P149 IMPLEMENTING TARGETED MOLECULAR RESISTANCE TESTING FOR GONORRHOEA IN A LARGE URBAN UK CLINIC}

${ }^{1} E$ Moss, ${ }^{1} A$ Maxwell, ${ }^{2} E$ Goldstein, ${ }^{2} \mathrm{R}$ Gunson, 'A Winter. 'Sandyford Sexual Health Service, NHS Greater Glasgow and Clyde, Glasgow, UK; ${ }^{2}$ West of Scotland Specialist Virology Centre, Glasgow, UK

10.1136/sextrans-2021-sti.257

Background Sandyford Sexual Health serves a population of ca. 1 million residents of Greater Glasgow and Clyde, UK. We use RealTime CT/NG assay (Abbott Molecular) to exclude gonorrhoea, all positive results are centrally notified and managed. We report our experience implementing 'resistanceguided' treatment for gonorrhoea using the SpeeDx ResistancePlus ${ }^{\circledR}$ GC test. The objective was to measure the proportion of patients successfully managed with oral rather than injectable treatment.
Methods Resistance testing was targeted at patients who might benefit, excluding patients who had empirical treatment. This was determined by our laboratory scientist who reviewed the electronic prescribing record. Tests were run twice weekly. Meaningful test name and results messages were designed to address anticipated human factors in assimilating the information.

Results Between 16th October and 24th December 2020, 93 patients had a positive gonorrhoea test meriting treatment, of which $20(22 \%)$ had infection at more than one anatomical site. Empirical treatment was given to 36 patients and so did not undergo resistance testing; 3 had insufficient residual sample. In the remaining 54 patients, 3 were gonorrhoea negative via SpeeDx ResistancePlus ${ }^{\circledR}$ testing and 2 were gyrA indeterminate. Ultimately, 33 had predicted ciprofloxacin sensitivity; 16 had predicted ciprofloxacin resistance.

Of the 33 patients identified as ciprofloxacin sensitive, 16 received oral ciprofloxacin and 17 received alternative standard-of-care. Reasons for not using ciprofloxacin included: clinician preference for injectable, resistance results not being available in time and quinolone contraindication.

No patients had discordant resistance predictions at different anatomical sites.

Conclusions Targeted molecular resistance testing for gonorrhoea averted injectable treatment in $17 \%$ of this cohort with around only half being eligible for testing due to immediate or prior treatment. We also averted some injections amongst contacts. Future work will focus on building clinician confidence and improving turnaround time in a production environment.

\section{P150 CHARACTERIZATION OF PHARYNGEAL GONORRHEA IN UGANDAN MEN WITH URETHRAL DISCHARGE SYNDROME}

${ }^{1} \mathrm{~J}$ Melendez ${ }^{*},{ }^{2} \mathrm{E}$ Mande, ${ }^{2} \mathrm{~A}$ Onzia, ${ }^{3} \mathrm{P}$ Kyambadde, ${ }^{2} \mathrm{M}$ Lamorde, ${ }^{1} \mathrm{Y}$ Manabe, ${ }^{1} \mathrm{M}$ Hamill. ${ }^{1} J o h n s$ Hopkins School of Medicine, Baltimore, USA; ${ }^{2}$ Infectious Diseases Institute, Makerere University, Kampala, Uganda; ${ }^{3}$ Ministry of Health, STI Control Unit, Kampala, Uganda

\subsection{6/sextrans-2021-sti.258}

Background Multi-drug resistant Neisseria gonorrhoeae (NG) threatens the effectiveness of gonorrhea treatment and control globally. Pharyngeal NG (pNG) infections are hypothesized to play a role in the emergence of antimicrobial resistance (AMR) in NG, yet the epidemiology of pNG infections is poorly understood, especially in resource-limited settings (RLS). We report on pNG in men with concurrent urogenital NG infections in Kampala, Uganda.

Methods Penile-meatal and pharyngeal samples were collected from Ugandan men with urethral discharge syndrome (UDS), cultured for NG, and tested by nucleic acid amplification tests (NAATs) for NG and other STIs. Antimicrobial susceptibility testing was performed on all NG isolates.

Results Of the 83 participants (mean age 29.5 years) who provided urogenital and pharyngeal samples, 22.9\% (19/83) were living with HIV, and $57.8 \%$ (48/83) were positive for urogenital gonorrhea. NG was detected in $8.6 \%$ (7/81) of pharyngeal samples by NAAT; $57.1 \%$ (4/7) were also positive by culture. Participants with pNG had concurrent urogenital NG, were HIV-negative, heterosexual (71.4\%), reported transactional sex 
$(85.7 \%)$, did not report oral sex $(85.7 \%)$, and were younger (median -24 years, IQR $=21-28 ; \mathrm{p}=0.013$ ) than pNG-negative participants (median -29 years, $\mathrm{IQR}=27-32$ ). The paired $(n=4)$ urogenital-pharyngeal isolates displayed the same antimicrobial susceptibility profile: ciprofloxacin-, tetracycline-, and penicillin-resistant and ceftriaxone- and cefixime-susceptible. None of the pharyngeal samples were positive for Chlamydia trachomatis and one sample was positive for Mycoplasma genitalium.

Conclusions To our knowledge, this is the first report of pNG in Ugandan men. NAATs were more sensitive than cultures for detection of pNG. In this pilot study, there was no difference in the susceptibility profiles of paired pharyngeal and urogenital NG isolates. Differences by age might be explained by differences in sexual behaviors or mucosal immunity. Additional studies are warranted to better define the epidemiology and microbiology of pNG infections in RLS.

\section{P151 SYSTEMATIC LITERATURE REVIEW AND QUANTITATIVE ANALYSIS OF HEALTH PROBLEMS ASSOCIATED WITH SEXUALLY TRANSMITTED NEISSERIA GONORRHOEAE INFECTION}

1J Whelan*, ${ }^{2} \mathrm{~J}$ Eeuwijk, ${ }^{2}$ E Bunge, ${ }^{3}$ E Beck. ${ }^{1}$ GSK Vaccines, Amsterdam, The Netherlands; ${ }^{2}$ Pallas health research and consultancy, Rotterdam, The Netherlands; ${ }^{3}$ GSK Vaccines, Wavre, Belgium

\subsection{6/sextrans-2021-sti.259}

Background Neisseria gonorrhoeae $(\mathrm{Ng})$ is the second most common sexually transmitted bacterial infection (STI), leading to serious health problems in men, women and newborns. While early antibiotic treatment is effective, infections are increasingly antibiotic-resistant. No systematic reviews present health problems associated with $\mathrm{Ng}$ infections or their likelihood of occurrence. The objective, therefore, was to conduct a systematic literature review to address these gaps.

Methods A systematic literature review was conducted of all studies with an English abstract published since 1950 (Pubmed)/1966 (Embase). The search included patients with a history of/current sexually transmitted $\mathrm{Ng}$ infection. Expected outcomes were defined from published reviews of gonorrhoea health problems. Observational studies with a control group were included. A decision tree determined the best quality studies for each outcome, prioritising generalisable populations, lab-confirmed diagnosis, clearly defined outcomes, no STI coinfections, adjusted analyses, and risk estimates. Where feasible, a meta-analysis was performed, otherwise the best quality study estimates were identified.

Results In total, 46 studies were included, and 22 health problems were identified. Of these problems, $\mathrm{Ng}$ infection was statistically significantly associated with preterm premature ruptures of membranes, preterm birth, low birth weight, stillbirth, infant death, neonatal ophthalmia, schizophrenia in offspring, pelvic inflammatory disease and subsequent tubal infertility, human immunodeficiency virus, and prostate cancer/ problems. High-quality evidence was generally lacking, with high heterogeneity across studies, and limited or inconclusive data on other health problems.

Conclusion $\mathrm{Ng}$ infection is associated with severe health problems in women, men and newborns. More high-quality comparative studies are needed to address the limitations in current knowledge.

\section{P152 COMMUNITY-BASED HIV TESTING IN THE NETHERLANDS: EXPERIENCES OF LAY PROVIDERS AND END USERS AT A RAPID HIV TEST CHECKPOINT}

E Op de Coul, R Spijker, A Żakowicz, M de Moraes, T Heijman, E Op de Coul*. Rivm, Bilthoven, The Netherlands

\subsection{6/sextrans-2021-sti.260}

Background The AIDS Healthcare Foundation (AHF-Checkpoint) in the Netherlands provides free 'walk-in', rapid HIV testing for key populations at onsite and pop-up locations. We explored the perspectives, experiences, and needs of lay providers and end users of this HIV test service, taking into account the WHO 5Cs (consent, confidentiality, counselling, correct results, connection-to-care) performance criteria for HIV test services.

Methods A qualitative evaluation with 15 semi-structured indepth interviews (April-June 2020) by telephone or video calling with ten lay providers and five end users from AHFCheckpoint. Recorded interviews were transcribed verbatim and thematically analysed.

Results Data analysis identified four domains: 1) accessibility of HIV testing, 2) quality of test procedures, 3) bridging (transitional care), and 4) future strategies for service delivery. AHF-Checkpoint fills a gap for key populations, including LGBTQ and refugees, who experience barriers to HIV testing at sexual health centres or GPs by providing anonymous, rapid testing. The level of trust between lay providers and end users was highly valued by end users. They also appreciated the low threshold to test, no waiting lists, no test costs or triaging that could include referral to another test location. Needs expressed by lay providers included more preparedness for emotionally charged situations and extra training to improve knowledge on STIs. Some end users expressed a need for a full STI test package at AHF-Checkpoint. Of the $5 \mathrm{Cs}$, consent, counselling, and correct results were realised, but confidentiality was sometimes difficult to achieve at popup locations, and referral barriers for confirmation testing (connection-to-care) were occasionally experienced by lay providers during weekends.

Conclusion AHF-Checkpoint was described as a convenient and easily accessible service by end users and lay providers. Of the WHO 5Cs, connection-to-care could be optimised to ensure HIV confirmation -and STI testing through a liaison approach with professionals from the regular healthcare sector.

\section{P155 CLINICAL PRESENTATION OF INCIDENT SYPHILIS AMONG MEN WHO HAVE SEX WITH MEN TAKING HIV PRE-EXPOSURE PROPHYLAXIS IN MELBOURNE, AUSTRALIA}

${ }^{1} \mathrm{~J}$ Peel* ${ }^{*},{ }^{1,2,3}{ }^{3}$ E Chow, ${ }^{1}$ I Denham, ${ }^{1} T$ Schmidt, ${ }^{1} \mathrm{~A}$ Buchanan, ${ }^{1,2} \mathrm{C}$ Fairley, ${ }^{4,5,6} \mathrm{D}$ Williamson, ${ }^{1} \mathrm{M}$ Bissessor, ${ }^{1,2} \mathrm{M}$ Chen. ${ }^{1}$ Melbourne Sexual Health Centre, Melbourne, Australia; ${ }^{2}$ Central Clinical School, Monash University, Melbourne, Australia; ${ }^{3}$ Centre for Epidemiology and Biostatistics, Melbourne School of Population and Global Health, The University of Melbourne, Melbourne, Australia; ${ }^{4}$ Microbiological Diagnostic Unit Public Health Laboratory, Department of Microbiology and Immunology, The University of Melbourne at The Peter Doherty Institute for Infection and Immunity, Melbourne, Australia; ${ }^{5}$ Department of Microbiology and Immunology, The University of Melbourne at The Peter Doherty Institute for Infection and Immunity, Melbourne, Australia; ${ }^{6}$ Department of Microbiology, Royal Melbourne Hospital, Melbourne, Australia

10.1136/sextrans-2021-sti.261 